\title{
A!
}

This is an electronic reprint of the original article.

This reprint may differ from the original in pagination and typographic detail.

Sheikh, Muhammad Usman; Jantti, Riku; Hamalainen, Jyri

\section{Impact of Interference Suppression under Ray Tracing and 3GPP Street Canyon Model}

Published in:

2020 IEEE 91st Vehicular Technology Conference, VTC Spring 2020 - Proceedings

DOI:

10.1109/VTC2020-Spring48590.2020.9129448

Published: 01/05/2020

Document Version

Peer reviewed version

Please cite the original version:

Sheikh, M. U., Jantti, R., \& Hamalainen, J. (2020). Impact of Interference Suppression under Ray Tracing and 3GPP Street Canyon Model. In 2020 IEEE 91st Vehicular Technology Conference, VTC Spring 2020 -

Proceedings [9129448] (IEEE Vehicular Technology Conference). IEEE. https://doi.org/10.1109/VTC2020Spring48590.2020.9129448

This material is protected by copyright and other intellectual property rights, and duplication or sale of all or part of any of the repository collections is not permitted, except that material may be duplicated by you for your research use or educational purposes in electronic or print form. You must obtain permission for any other use. Electronic or print copies may not be offered, whether for sale or otherwise to anyone who is not an authorised user. 


\title{
Impact of Interference Suppression under Ray Tracing and 3GPP Street Canyon Model
}

\author{
Muhammad Usman Sheikh, Riku Jäntti and Jyri Hämäläinen \\ Department of Communications and Networking \\ Aalto University \\ 02150 Espoo, Finland \\ Email: \{muhammad.sheikh, riku.jantti and jyri.hamalainen\}@aalto.fi
}

\begin{abstract}
Channel models are routinely used for evaluating the performance of wireless technologies and cellular networks. An appropriate channel model is necessary for a credible system analysis, and the shortcomings in the channel model may lead to erroneous conclusions. In this paper we characterize the impact of Interference Suppression (IS), Interference Cancellation (IC) and interference management on the system performance when using a) the 3rd Generation Partnership Project (3GPP) street canyon model (TR 38.901, Release 14), b) Shoot and Bouncing Ray (SBR) method based Ray Tracing (RT) model, and c) a proposed large scale path loss model in an urban, so-called Manhattan building grid environment. Simulations are performed using the $28 \mathrm{GHz}$ carrier frequency that has been recently considered for the $5^{t h}$ Generation (5G) networks. Simulation results indicate that the 3 GPP channel model provides slightly pessimistic path loss values than RT in Line of Sight (LOS) conditions, whereas in Non-LOS (NLOS) situation it gives a considerable pessimistic path loss estimation as compared with the deterministic RT approach. The difference between channel models is notable especially for the estimation of Signal to Interference plus Noise Ratio (SINR). Since RT provides realistic results due to accurate radio environment and signal modeling and, on the other hand, the correct SINR estimation is crucial for the wireless system evaluation. Our proposed path loss model is based on RT simulations. The performance of the proposed model for different performance metrics matches well with the RT results.
\end{abstract}

Index Terms-Interference; Ray tracing; 3GPP; 5G; System performance; Simulations.

\section{INTRODUCTION}

The rapid increase of mobile data users with different service requirements makes a demand for an active development of cellular networks. Base station (BS) site densification is found as an elementary and straightforward way of increasing the system capacity, see e.g. [1]. However, dense cellular networks suffer from Inter-Cell Interference (ICI). The reuse of frequency resources could solve the interference problem, but the frequency spectrum is expensive and is already in extensive use. Therefore, the inefficient use of core resources is not a practical option and the ICI is imperative. The level of interference in the cellular networks depends upon numerous factors such as the network layout, propagation environment, frequency reuse, transmission power, and multiple access techniques [2]. The problem of ICI becomes even more severe in the so-called Ultra Dense Network (UDN), due to highly dense deployment of base statiions with small inter-site distance.
Several techniques have been developed to avoid and to mitigate the ICI. These techniques include, for example, beamforming [3], frequency domain Intercell Interference Coordnation (ICIC), Fractional Frequency Reuse (FFR) [4], Enhanced ICIC (eICIC) and Further enhanced ICIC (FeICIC) , Coordinated Multipoint Transmission (CoMP) [5], [6], power control and, in addition, advanced receivers may employ e.g. Interference Rejection Combining (IRC) [7] or Network Assisted Interference Cancellation and Suppression (NAICS) [8]. The target of these techniques is to improve the spectrum efficiency and to support higher data rates.

Research community has made a huge effort while developing and evaluating the above-mentioned techniques. Results from performance evaluations are important since they impact on the standardization and product development decisions. Performance evaluations are largely carried out through computer simulations. To enable the drawing of credible conclusions, simple yet accurate channel models are needed. Accordingly, the $3^{\text {rd }}$ Generation Partnership Project (3GPP) have proposed several channel models for different radio environments in order to evaluate the cellular systems performance at frequencies ranging from $0.5 \mathrm{GHz}$ to $100 \mathrm{GHz}$ [9].

Besides typically applied channel models that rely on averaged channel measurements, there is a deterministic approach of Ray Tracing (RT) [10]. Literature shows two broad classes of ray tracing algorithms based on their implementation i.e. Image Theory (IT) [11] and Shoot and Bouncing Ray (SBR) method [12], [13], also known as Ray Launching (RL) method. Available ray tracing models are credible and accurate [14], [15]. The ray tracing simulation results depend on case-specific 3D propagation environment whereas, the 3GPP models are based on averaged channel data. Ray tracing simulations are computationally complex, the load and the level of computation increases with the complexity of the simulation environment. We recall that it is not well-known whether RT and 3GPP models provide comparable results.

In this paper we compare the 3GPP street canyon model (TR 38.901, Release 14) and SBR method based RT model in an urban, so-called Manhattan building grid environment. We assume $28 \mathrm{GHz}$ carrier frequency that has been recently considered for the $5 \mathrm{G}$ networks. Results show that the $3 \mathrm{GPP}$ channel model provides slightly more pessimistic path loss values than RT in Line of Sight (LOS) conditions. Whereas, 
in Non-LOS (NLOS) situation 3GPP model proposes clearly higher path loss values than the deterministic RT approach. This difference between channel models is notable while estimating the Signal to Interference plus Noise Ratio (SINR). Furthermore, the overoptimistic SINR from the 3GPP model leads to a considerably higher throughput expectations. This may even lead to erroneous system performance conclusions. Since RT provides realistic results due to accurate radio environment and signal modeling and, on the other hand, the correct SINR estimation is crucial for the wireless system evaluation, we propose a new path loss model based on the RT results. The SINR and throughput performance of the proposed model matches well with the RT results.

\section{BACKGROUND THEORY}

\section{A. Ray Tracing (RT) - Shoot and Bouncing Ray (SBR)}

Nowadays, ray tracing methods have gained a lot of interest due to the easy availability of servers and clusters with high computational capabilities. Ray tracing methods find the LOS path along with other propagation paths with reflections, diffractions, and transmission/penetration. The Image Theory (IT) method [11] and the Shoot and Bouncing Ray (SBR) method [12], [13] also known as Ray Launching (RL) method are the two well known methods available in literature for ray tracing. In this study we employ SBR method for simulations. In SBR, a finite number of rays are emitted from the transmitter assuming a certain discrete separation in angular domain. The launched rays continue to propagate until they are obstructed or they fall on some wall. The rays are launched in omni directions, however, only a small fraction of the rays reach the receiver due to blockage and geometry of environment. The received power is the sum/composite of the multiple rays coming from different directions [12]. The computational load of the SBR increases with the increase in the number of emitted rays, and also depends upon the geometry of the simulation environment [11], [13].

\section{B. 3GPP Street Canyon Pathloss Model for Urban Microcel- lular Environment (UMi)}

3GPP provides environment specific channel models in Technical Report TR 38.901 Release 14 for frequencies ranging from $0.5 \mathrm{GHz}$ to $100 \mathrm{GHz}$ [9]. The channel models presented in [9] are applicable for both link level and system level simulations, and are valid for Rural Macrocellular $(R M a)$, Urban Macrocellular (UMa), and Urban Microcellular (UMi) environment. We mainly focus on the urban microcellular regular building grid environment considering outdoor base station serving outdoor users, therefore only Outdoor to Outdoor $(\mathrm{O} 2 \mathrm{O}) U M i$ case is studied here.

For a communication link with transmit power $P_{T}$, Transmitter (TX) antenna gain $G_{T}$ and Receiver (RX) antenna gain $G_{R}$, the received power $P_{R}$ in $d B m$ at the receiver point is equal to $P_{T}[d B m]+G_{T}[d B i]+G_{R}[d B i]-P L[d B]$, where $P L$ denotes the large scale radio propagation Pathloss (PL) [16]. Herein, the pathloss is a function of carrier frequency and distance between the RX and TX. In $U M i$ case, for TXRX separation smaller than the so-called breakpoint distance, the large scale pathloss model in LOS condition is given by Eq. 1. The 3GPP framework also offers an optional pathloss model without considering the RX height as given in Eq. 2.

$$
\begin{gathered}
P L_{L O S}=32.4+20 \log _{10}\left(f_{c}\right)+21 \log _{10}\left(d_{3 D}\right) \\
P L_{N L O S}=32.4+20 \log _{10}\left(f_{c}\right)+31.9 \log _{10}\left(d_{3 D}\right)
\end{gathered}
$$

We note that in Eq. 1 and Eq. 2, $f_{c}$ is the carrier frequency of operation in $[\mathrm{GHz}]$, and $d_{3 D}$ is the $3 \mathrm{D}$ distance between the transmitter and the receiver in meters $[m]$. The simulation scenarios discussed in this paper only involve distances smaller than the breakpoint distance. Therefore, we apply the pathloss models presented in Eq. 1 and Eq. 2 for simulation purposes.

\section{Proposed Large Scale Pathloss Model for Street Canyon (Manhattan) Environment}

The large scale pathloss model for microcellular environment presented by 3GPP in [9] is based on the measurements done in a specific environment. However, a widely applied homogeneous building grid environment used in system performance simulations is different from the scenario where the actual measurements were carried out. Therefore, the 3GPP $U M i$ pathloss model may provide erroneous results. To fix this shortcoming, we propose a large scale PL model based on the ray tracing simulation results. Here, curve fitting algorithm with linear regression is used over the ray tracing data to obtain the proposed model parameters. The proposed model considers the same parameters and follows the same format as used in the 3GPP model. However, the values of the coefficients were adjusted after the curve fitting. The new model for microcelluar street canyon environment with LOS and NLOS condition is given in Eq. 3 and Eq. 4, respectively.

$$
P L_{L O S}=34+20 \log _{10}\left(f_{c}\right)+21.82 \log _{10}\left(d_{3 D}\right)
$$

$$
P L_{N L O S}=30+20 \log _{10}\left(f_{c}\right)+26.5 \log _{10}\left(d_{3 D}\right)
$$

\section{Simulation Setup, Models and Parameters}

\section{A. Simulation Scenario and Tools}

For deterministic ray tracing simulations a homogeneous regular building grid is considered as illustrated in Fig. 1. Each square represents a chunk of building blocks covering $100 \mathrm{~m} \times 100 \mathrm{~m}$ area. The height of the buildings is $30 \mathrm{~m}$, and there is a $30 \mathrm{~m}$ wide road between two successive building blocks. In the applied micro-cell deployment there are nineteen sites, and each site has three micro-cells. The three cells in each micro site have an azimuth of $0^{\circ}, 135^{\circ}, 225^{\circ}$. Antennas of the micro-cells are placed on the outer wall of the building at 25 meter height. The maximum transmission power of microcells is set to $33 \mathrm{dBm}$ ( 2 watts). There is no power control 


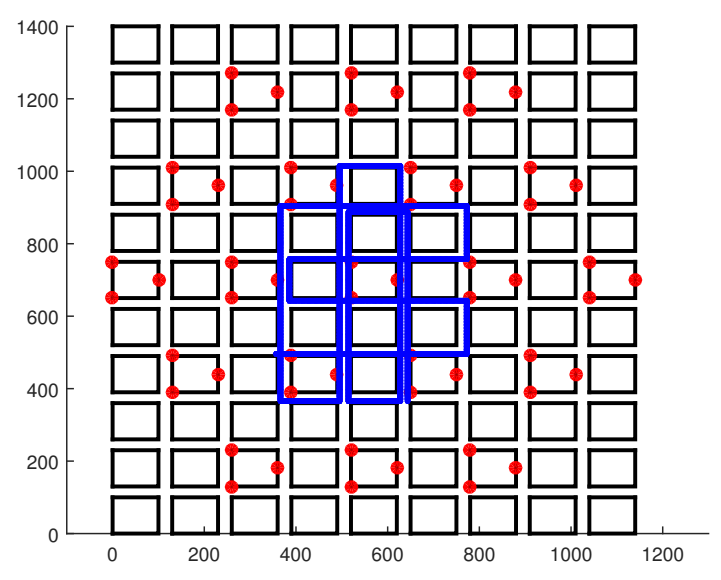

Fig. 1. Illustration of grid environment along with location of micro cells.

and all cells transmit with their full power irrespective of the cell load. For 3GPP and proposed model simulations the same location of cells are used, and the map data is needed only to extract the information about the existence of LOS or NLOS between TX and RX. A receiver with $1.6 \mathrm{~m}$ height and $3.6 \mathrm{~m} / \mathrm{s}$ speed follows the simulation route in an outdoor environment as shown by the blue line in Fig. 1.

MATLAB is used as a simulation platform, and a $3 \mathrm{D}$ ray tracing/launching tool based on SBR method is developed by the authors in MATLAB. This RT tool finds the propagation paths between the TX and RX considering the 3D geometry of the environment, and the position of the TX and RX. The RT tool not only finds the LOS path, but it also provides the paths with multiple reflections and diffractions and the penetrated path through the buildings. The ray tracing tool launches the rays with $0.5^{\circ}$ angular separation from the transmitter point. In a street canyon environment, it is critical to consider the diffracted paths coming from the edges/corners of the buildings, especially the paths with single diffraction. The maximum number of supported reflections and diffractions in our ray tracing simulation is set to ten and one, respectively. A penetrated path is the direct path between the TX and RX passing through the buildings in NLOS case.

\section{B. Additional Simulation Models}

Building Penetration Loss (BPL) is taken into account for the penetrated path. The BPL is modeled as a function of the carrier frequency and the material of the wall/building. The Table 7.4.3-1 at [9] shows the penetration loss for different material types. Two penetration loss models, low-loss model and high-loss model for old and modern building wall type, respectively, are presented in Table 7.4.3-2 of [9]. We have considered a high-loss model in our ray tracing simulations.

Large scale fading, also known as shadow fading, is typically modeled by using the log-normal distribution in decibel scale. The standard deviations of shadow fading for different environment types in LOS/NLOS scenario are given in Table 7.4.1-1 of [9]. We have considered the mobility of the users
TABLE I

3GPP ANTENNA MODEL PARAMETERS

\begin{tabular}{|c|c|c|c|c|}
\hline $\begin{array}{c}\theta_{H} \\
{\left[^{\circ}\right]}\end{array}$ & $\begin{array}{c}\theta_{V} \\
{\left[^{\circ}\right]}\end{array}$ & $\begin{array}{c}F B R_{H} \\
{[d B]}\end{array}$ & $\begin{array}{c}S L L_{V} \\
{[d B]}\end{array}$ & $\begin{array}{c}A_{M} \\
{[d B i]}\end{array}$ \\
\hline 65 & 7 & 30 & -18 & 17.7 \\
\hline
\end{tabular}

along the simulation route where shadowing is correlated. Correlated shadowing between successive points is given by normalized auto-correlation function in section 7.4.4 of [9].

Finally, the antenna radiation pattern of the directional antenna used at the BS is modeled by using 3GPP antenna model presented in [9]. Antenna radiation modeling parameters i.e. Half Power Beamwidth (HPBW) in horizontal $\left(\theta_{H}\right)$, HPBW in vertical domain $\left(\theta_{V}\right)$, Front to Back Ratio in azimuth plane $\left(F B R_{H}\right)$, Side Lobe Level in elevation plane $\left(S L L_{V}\right)$, and antenna maximum gain $\left(A_{M}\right)$ are provided in Table. I.

\section{Performance metrics}

The system performance is analyzed in terms of coverage i.e. received power, quality i.e. Signal to Interference plus Noise Ratio (SINR), and user experience i.e. user application layer throughput. In order to depict a real user experience, the impact of user mobility (handover) is taken into account while analyzing different performance metrics. The Handover Cost $\left(\mathrm{HO}_{\text {Cost }}\right)$ is a function of Handover Rate $\left(\mathrm{HO}_{\text {Rate }}\right)$ and Handover Delay $\left(H O_{\text {Delay }}\right)$, and it is given by Eq. 5. A handover delay of $2 \mathrm{~s}$ is applied in our simulations.

$$
H O_{\text {Cost }}=H O_{\text {Rate }} * H O_{\text {Delay }}
$$

The SINR to application layer user throughput mapping is carried out by an approximation based on the modified form of Shannon's capacity formula as presented in Eq. 6.

$$
T=N_{t}\left(B * \log _{2}(1+S I N R)\right)\left(1-H O_{C o s t}\right)(1-\alpha)
$$

In Eq. 6, $N_{t}$ is the number of transmit antennas or independent bit streams, $B$ is the system bandwidth, and $\alpha$ is the control overhead. A $30 \%$ control overhead is assumed in our simulations.

\section{Simulation Results and Discussion}

This section provides comprehensive simulation results for the ray tracing, 3GPP model and the proposed model. Fig. 2 shows the Cumulative Distribution Function (CDF) of the received signal power. Results in Fig. 2 are based on 20 snapshots of Monte Carlo simulations along the simulation route. The 3GPP model is found as the most optimistic while it provides the mean received signal strength of $-69.3 \mathrm{dBm}$, whereas RT and proposed model offers the mean received power of $-72.3 \mathrm{dBm}$ and $-72.6 \mathrm{dBm}$, respectively. We emphasize that after analyzing the simulation data it was found that, due to the dense deployment of micro-cells, 98.8-99.5\% of the simulation route locations admit a LOS with the serving base station. Hence, the main contribution in results is coming from the 3GPP-LOS and the proposed LOS models, where the 
3GPP model yields $3 \mathrm{~dB}$ better mean received power when compared with the RT. At the same time, the proposed model provides $0.3 \mathrm{~dB}$ less power when compared with RT.

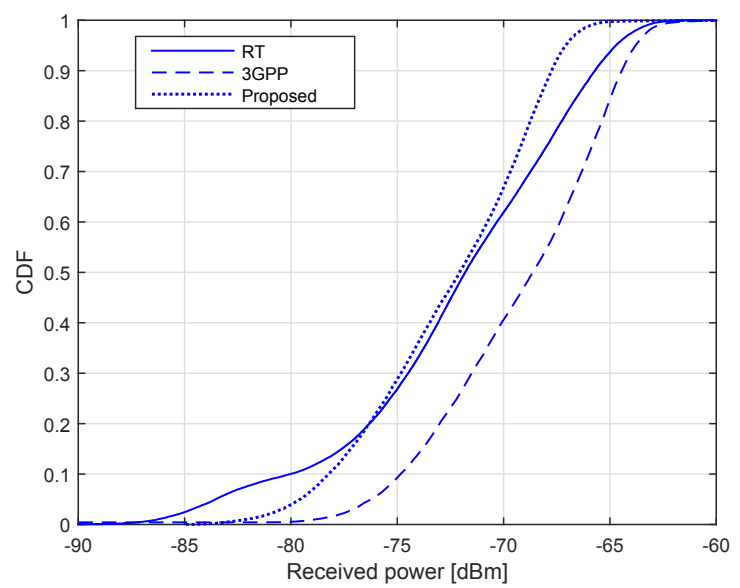

Fig. 2. CDF plots of received signal strength.

The Fig. 3 shows the CDF of received interference power. It can be seen that the top 26 percentile samples are almost identical for the 3GPP and the proposed model. Interestingly, the 3 GPP model underestimates the pathloss in Fig. 2 but it overestimates the PL of interferers in Fig. 3 as compared to RT model. This is due to fact that in results of Fig. 2 the main contribution is coming from the LOS model while the interference is arriving through both LOS and NLOS signal paths.

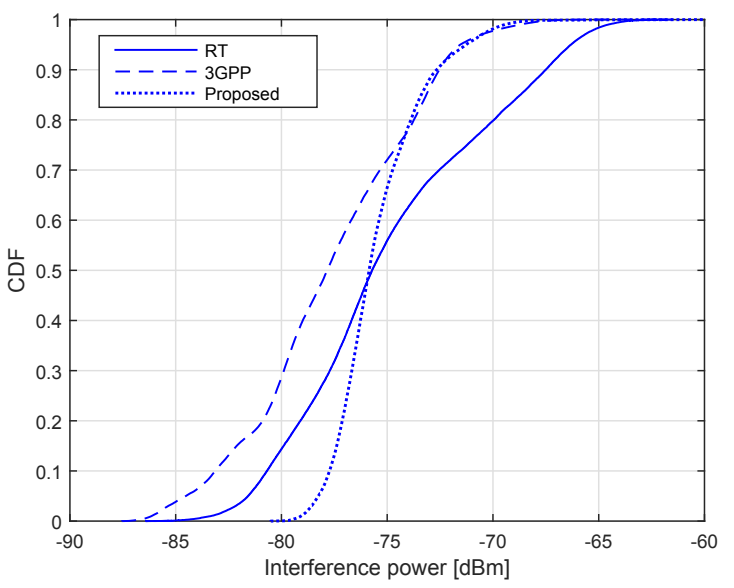

Fig. 3. CDF plots of received interference power.

Table II shows the LOS percentages of top three interferers. The numbers presented in Table II show that the interference behaves differently in three different models. As mentioned earlier, 3GPP LOS model underestimates the pathloss, and at the same time there is a high probability for the strongest and the second strongest interferer to be in LOS with the receiver. Whereas, the proposed model relaxes the pathloss for NLOS case, and there is a comparatively small chance for a LOS interference. The mean RX interference power given by $3 \mathrm{GPP}$ model is almost $2.5 \mathrm{~dB}$ less as compared with the RT and the proposed model. However, the mean received interference power is pretty close for the RT and the proposed model.

TABLE II

LOS PERCENTAGE OF TOP THREE INTERFERERS

\begin{tabular}{l|c|c|c}
\hline Cases & $\begin{array}{c}1^{\text {st }} \text { Interferer } \\
{[\%]}\end{array}$ & $\begin{array}{c}2^{\text {nd }} \text { Interferer } \\
{[\%]}\end{array}$ & $\begin{array}{c}3^{\text {rd }} \text { Interferer } \\
{[\%]}\end{array}$ \\
\hline 3GPP & 96.4 & 75.8 & 67.7 \\
RT & 77.2 & 78.5 & 67.2 \\
Proposed & 32.9 & 45.2 & 25.7 \\
\hline
\end{tabular}

The interference contribution of the strongest interferer along with the second strongest and third strongest interferer is shown in Fig. 4. The plots of Fig. 4 indicate that in case of $3 \mathrm{GPP}$ and RT models, the major portion of interference is coming from the top three interferers. However, in case of the proposed model the interference arrives more evenly from larger number of sources. Fig. 4 shows that the strongest interferer alone contributes $63.5 \%, 57 \%$, and $49 \%$ of the total interference level in case of 3GPP, RT, and proposed models, respectively. Furthermore, Fig. 4 shows that top three interferers constitute to almost $90-92 \%$ of the total intereference in case of 3GPP and RT models, while for the proposed model round $77 \%$ of the interference comes from the top three interferers. Therefore, it can be concluded that by suppressing or avoiding the top one or two interferers we can significantly improve the signal quality.

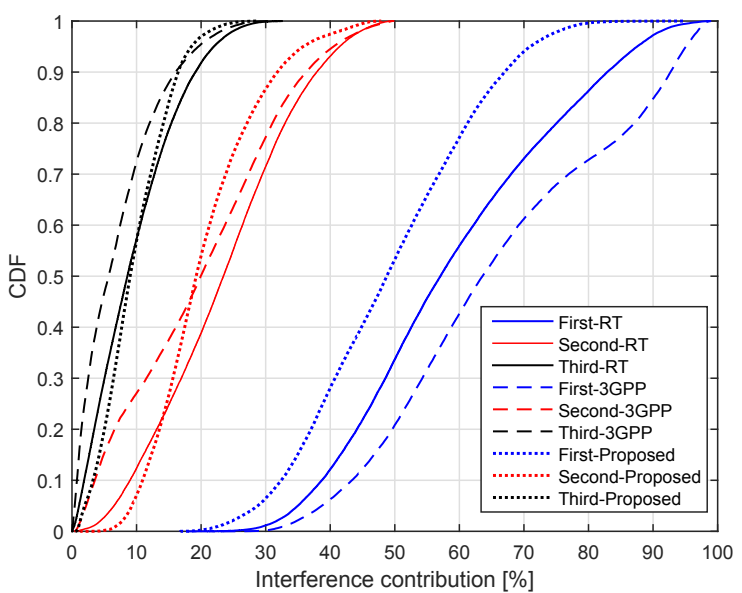

Fig. 4. CDF plots of interference contribution.

The quality metric is the downlink SINR. Fig. 5 shows the CDF plot of SINR with and without considering the interference suppression or cancellation. Without any interference suppression, the mean SINR value of around $8.4 \mathrm{~dB}$ is resulting from the $3 \mathrm{GPP}$ model, whereas the ray tracing and the proposed model offers a mean SINR of $2.6 \mathrm{~dB}$ and $2.91 \mathrm{~dB}$, respectively. The 3GPP model clearly overestimates the quality of the network and shows a difference of nearly $5.5 \mathrm{~dB}$ with the RT model. On the other hand the SINR obtained with the proposed model has a small difference of 


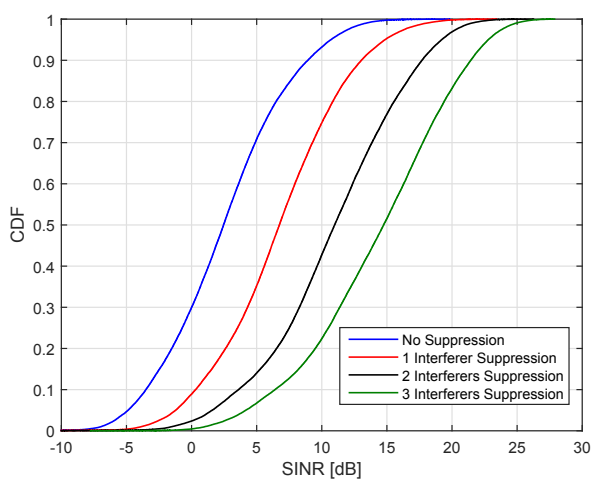

(a)

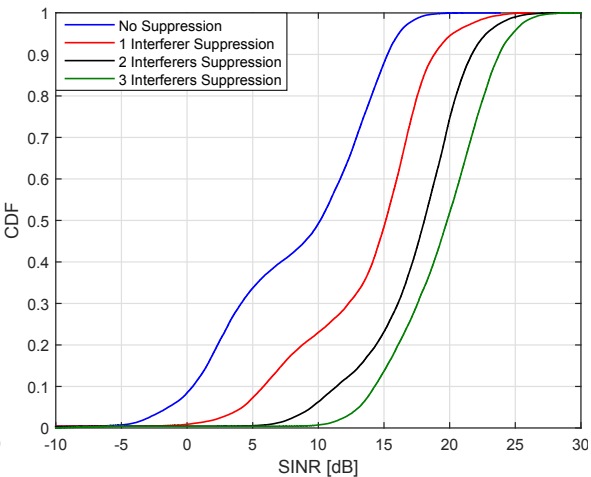

(b)

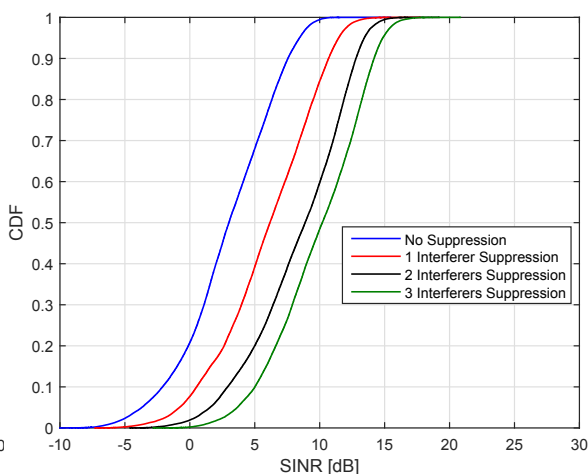

(c)

Fig. 5. CDF plots of SINR with, (a) Ray tracing model (b) 3GPP model, and (c) Proposed model.

around $0.31 \mathrm{~dB}$ compared with the RT model. It is also shown in Fig. 5(a-c) that the SINR can be significantly improved by completely suppressing the top interferer. In case of RT simulations, the improvement in SINR is almost linear up to three interferer suppression as shown in Fig. 5(a). Whereas, in the 3GPP and the proposed model the improvement in SINR gets smaller with the increasing number of interferer suppression. Also, it can be seen that in the proposed model case, as the interference is more distributed, the improvement in SINR is relatively small when compared with the RT and the 3GPP model.

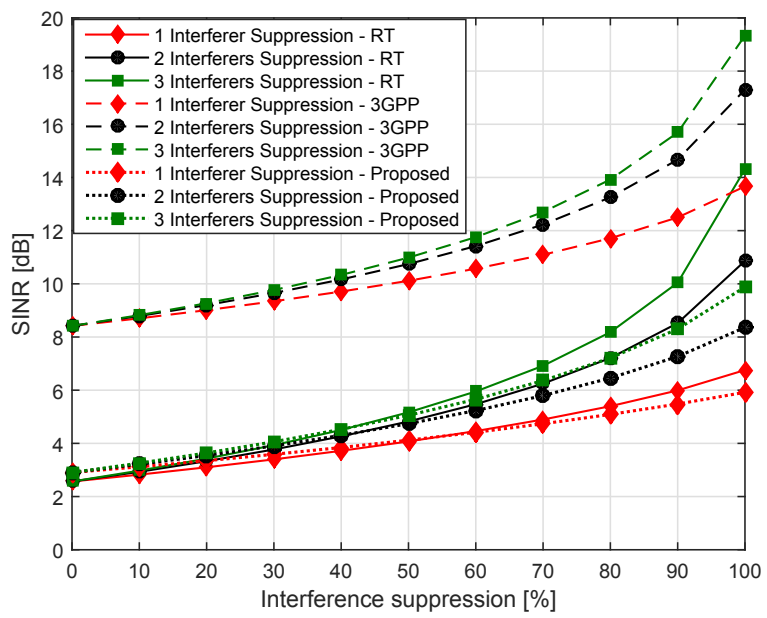

Fig. 6. SINR with interference suppression.

The results presented in Fig. 5 assume 100\% suppression of target interferers and that is hard to achieve in real life. Thus, in Fig. 6 we have provided the SINR when only a certain percentage of interference power is suppressed from the top three interterence contributors. The solid line shows the results for RT, the dashed line and the dotted line shows the results for $3 \mathrm{GPP}$ and the proposed model, respectively. It is seen that in case of the 3GPP model, the mean SINR can be improved by $1.7 \mathrm{~dB}$ just by suppressing $50 \%$ of the strongest interferer, whereas the RT and the proposed model shows the SINR improvement of $1.6 \mathrm{~dB}$. Similarly, the gain in SINR with different number of interferers and percentages of interference suppression can be seen in Fig. 6 .

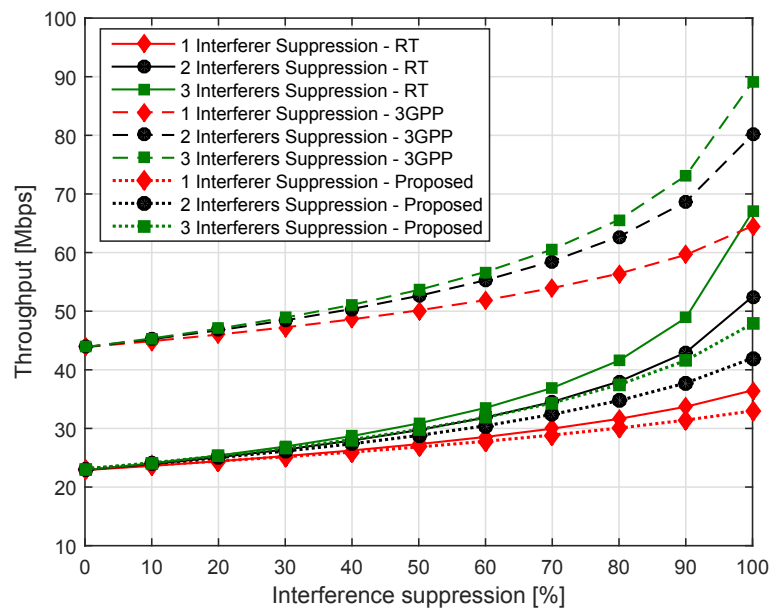

Fig. 7. Throughput with interference suppression.

As stated earlier, the SINR can be directly translated into throughput by using the formula given in Eq. 6. The Fig. 7 shows the throughput with and without considering the interference suppression percentage. The $3 \mathrm{GPP}$ model, RT model and the proposed model offers a mean throughput of 43.9 Mbps, 22.9 Mbps, and 23.1 Mbps, respectively, without considering any interference suppression. Results show a $5.5 \mathrm{~dB}$ difference in SINR for the 3GPP model and the RT model. That leads to a difference of $21 \mathrm{Mbps}$ in the user throughput. The 3GPP model also shows that user throughput can be improved from $43.9 \mathrm{Mbps}$ to $50 \mathrm{Mbps}$ by suppressing/rejecting $50 \%$ of the strongest interferer. Similarly, the RT model and the proposed model show the throughput improvement of about 26.8 Mbps with $50 \%$ rejection of the strongest interferer.

Fig. 8 shows the relative difference/change in the throughput of the 3GPP model and the proposed model with respect to the reference RT model. The 3GPP model provides $92 \%$ more optimistic throughput or in other words say erroneous result with respect to the RT model. The proposed model shows 


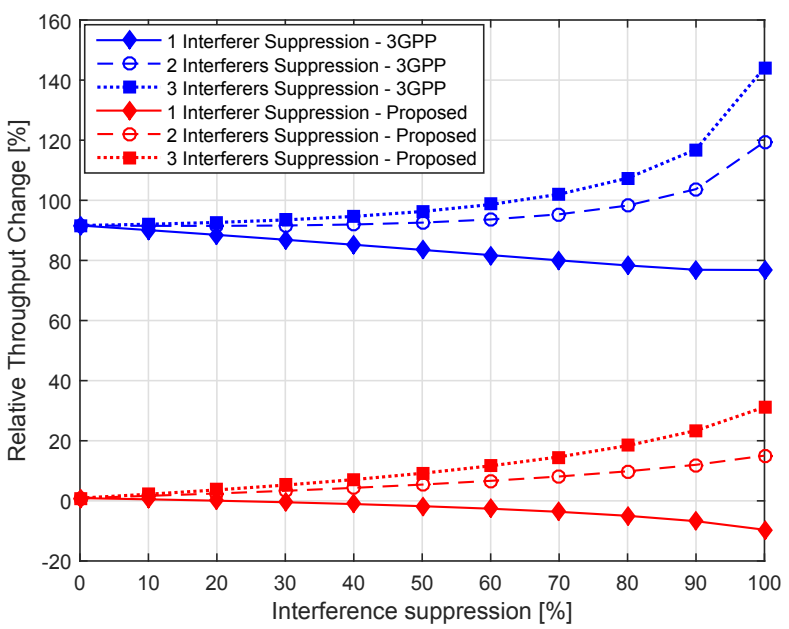

Fig. 8. Relative throughput change with respect to ray tracing model.

quite harmonized results with RT, and even with interference suppression the relative change in throughput is limited between $-10 \%$ to $30 \%$, whereas the $3 \mathrm{GPP}$ model may provide overestimated throughput upto $144 \%$.

\section{CONCLUSiON}

We highlighted the interference mitigation/suppression gain in dense homogeneous micro cellular network, specifically in a Manhattan grid environment. The presented results also emphasize the importance of the channel model accuracy in the system performance analysis. Results are based on comprehensive simulations performed at $28 \mathrm{GHz}$ using the 3GPP model, the ray tracing model and a newly proposed pathloss model. The performance of the 3GPP model and the RT model was compared from different perspectives, arising the question on the applicability of the 3GPP model in a microcellular street canyon environment.

In terms of received signal power from the serving base station, the 3GPP model is most optimistic and it provides nearly $3 \mathrm{~dB}$ better signal power levels compared with RT model. On the other hand, the $3 \mathrm{GPP}$ model provides the mean received interference power that is almost $2.5 \mathrm{~dB}$ lower as compared with the RT model and the newly proposed model. This leads to a difference of around $5.5 \mathrm{~dB}$ in the SINR between the 3GPP model and the RT model. The difference in SINR notably reflects to a user throughput: the 3GPP model provides even 21 Mbps higher throughputs than the RT model. Thus, the 3GPP model overestimates the user throughput by $92 \%$ with respect to the ray tracing approach, whereas the results acquired with newly proposed model are in harmony with the ray tracing simulations.

The simulation results clearly indicate that in the downlink direction, there is a need to attenuate the interference mainly coming from one or two neighbouring sources to improve the signal quality and the user experience by a healthy margin. The strongest interferer forms almost $63.5 \%, 57 \%$, and $49 \%$ of the mean interference level in case of 3GPP, RT, and proposed models, respectively. All the considered models showed that that the mean SINR can be improved by $1.6-1.7 \mathrm{~dB}$ only by suppressing the strongest interferer by $50 \%$.

\section{ACKNOWLEDGEMENT}

This work was partly supported by the Finnish public funding agency for research, Business Finland under the projects "5G Finnish Open Research Collaboration Ecosystem (5G-FORCE)" which is part of of 5G Test Network Finland (5GTNF), and by Academy of Finland under (grants No. 287249 and 311752).

\section{REFERENCES}

[1] D. Lopez-Perez, M. Ding, H. Claussen, and A. H. Jafari, "Towards $1 \mathrm{Gbps} / \mathrm{UE}$ in cellular systems: Understanding ultra-dense small cell deployments," IEEE Communication Surveys \& Tutorials, vol. 17, no. 4, pp. 2078-2101, Fourth Quarter 2015.

[2] Y. Zhou, L. Liu, H. Du, L. Tian, X. Wang, and J. Shi, "An overview on intercell interference management in mobile cellular networks: From $2 \mathrm{G}$ to 5G," in 2014 IEEE International Conference on Communication Systems, Nov 2014, pp. 217-221.

[3] W. Roh, J. Seol, J. Park, B. Lee, J. Lee, Y. Kim, J. Cho, K. Cheun, and F. Aryanfar, "Millimeter-wave beamforming as an enabling technology for $5 \mathrm{G}$ cellular communications: theoretical feasibility and prototype results," IEEE Communications Magazine, vol. 52, no. 2, pp. 106-113, February 2014.

[4] G. Boudreau, J. Panicker, N. Guo, R. Chang, N. Wang, and S. Vrzic, "Interference coordination and cancellation for 4G networks," IEEE Communications Magazine, vol. 47, no. 4, pp. 74-81, April 2009.

[5] R. Irmer, H. Droste, P. Marsch, M. Grieger, G. Fettweis, S. Brueck, H. Mayer, L. Thiele, and V. Jungnickel, "Coordinated multipoint: Concepts, performance, and field trial results," IEEE Communications Magazine, vol. 49, no. 2, pp. 102-111, February 2011.

[6] J. Lee, Y. Kim, H. Lee, B. L. Ng, D. Mazzarese, J. Liu, W. Xiao, and Y. Zhou, "Coordinated multipoint transmission and reception in LTEAdvanced systems," IEEE Communications Magazine, vol. 50, no. 11, pp. 44-50, November 2012.

[7] Y. Ohwatari, N. Miki, T. Asai, T. Abe, and H. Taoka, "Performance of advanced receiver employing interference rejection combining to suppress inter-cell interference in LTE-Advanced downlink," in 2011 IEEE Vehicular Technology Conference (VTC Fall), Sep. 2011, pp. 1-7.

[8] 3GPP, "Study on network-assisted interference cancellation and suppression (naic) for lte," 3rd Generation Partnership Project (3GPP), Technical Report (TR) 36.866, 03 2014, version 12.0.1.

[9] — "Study on channel model for frequencies from 0.5 to $100 \mathrm{ghz}$," 3rd Generation Partnership Project (3GPP), Technical Report (TR) 38.901, 12 2017, version 14.3.0.

[10] S. Hur, et al., "Proposal on millimeter-wave channel modeling for 5G cellular system," IEEE Journal on Selected Areas in Signal Processing, vol. 10, no. 3, pp. $454-469,2016$.

[11] S. Soni and A. Bhattacharya, "An efficient two-dimensional ray-tracing algorithm for modeling of urban microcellular environments," International Journal of Electronics and Communications, vol. 66, no. 6, pp. 439 - 447, June 2012.

[12] G. Durgin, N. Patwari, and T. S. Rappaport, "Improved 3D ray launching method for wireless propagation prediction," Electronics Letters, vol. 33, no. 16, pp. 1412-1413, July 1997.

[13] R. A. Kipp and M. C. Miller, "Shooting-and-bouncing ray method for 3D indoor wireless propagation in WLAN applications," in IEEE Antennas and Propagation Society Symposium, 2004., vol. 2, June 2004, pp. 1639-1642 Vol.2.

[14] B. Neekzad, K. Sayrafian-Pour, J. Perez, and J. S. Baras, "Comparison of Ray Tracing Simulations and Millimeter Wave Channel Sounding Measurements," in The 18th International Symposium on Personal, Indoor and Mobile Radio Communications, Sep. 2007, pp. 1-5.

[15] Z. Yun and M. F. Iskander, "Ray tracing for radio propagation modeling: Principles and applications," IEEE Access, vol. 3, pp. 1089-1100, 2015.

[16] T. Rappaport, R. Heath, R. Daniels, and J. Murdock, Millimeter wave wireless communications. Prentice Hall, 2015, includes bibliographical references (pages 585-651) and index. 